\title{
Use of contraceptive services in Britain: findings from the second National Survey of Sexual Attitudes and Lifestyles (Natsal-2)
}

\author{
Rebecca S French, Catherine H Mercer, Anne M Johnson, Kevin A Fenton, Bob Erens, Kaye Wellings
}

\begin{abstract}
Objective To describe contraceptive service use and identify demographic and sexual behavioural characteristics associated with use (and non-use) of different services.
\end{abstract}

Methods Probability survey sample of men and women aged 16-44 years, resident in Britain. Participants comprised 3369 men and 4375 women reporting vaginal intercourse in the last year (excluding those reporting exclusive use of sterilisation or medical investigations for infertility). Main outcome measures were use of contraceptive services, grouped as: general practice, community contraceptive clinics, retail services and nonuse of services.

Results General practice was the most commonly reported source of contraceptive supplies for women $(59.2 \%)$, while retail services were most frequently reported by men (42.7\%). $16.3 \%$ of women and $7.3 \%$ of men reported using more than one type of service. $20.7 \%$ of women and $45.1 \%$ of men had used no service

\section{Introduction}

Major advances in contraceptive service provision have been seen in Britain since Marie Stopes opened the first contraceptive clinic in 1921. During the inter-war period, the number of such clinics proliferated as the concept of 'family planning', with its emphasis on birth spacing and healthier mothers and children, gained increasing currency. In the late 1960 s and early 1970 s, contraceptive service provision was to extend more into general practice, with the availability of a medically prescribed and effective method, the contraceptive pill. ${ }^{1}$ The most recent development in service provision is the role of the retail sector in the provision of emergency contraception.

Centre for Sexual Health and HIV Research, Research Department of Infection and Population Health, University College London, London, UK

Rebecca French, MSc, Phd, Senior Research Associate

Catherine Mercer, MSc, PhD, Senior Research Fellow

Anne M Johnson, MD, F Med Sci, Professor

Division of STD Prevention, National Centers for HIV, STD and TB Prevention, Centers for Disease Control and Prevention (CDC), Atlanta, GA, USA, and Centre for Sexual Health and HIV Research, University College London, London, UK

Kevin Fenton, MD, PhD, Chief, National Syphilis Elimination Effort

National Centre for Social Research, London, UK

Bob Erens, BA, MA, Director, Survey Methods Unit

Centre for Sexual and Reproductive Health Research, Department of Public Health and Policy, London School of Hygiene and Tropical Medicine, London, UK Kaye Wellings, FRCOG, FFSRH, Professor

Correspondence to: Dr Rebecca French, Centre for Sexual Health and HIV Research, University College London, The Margaret Pyke Centre, 73 Charlotte Street, London W1T 4PL, UK. E-mail: rfrench@gum.ucl.ac.uk in the last year, and amongst 16-17-year-olds the proportions reporting non-use of services was $13.8 \%$ and $31.2 \%$, respectively. Use of community contraceptive clinics was associated with being younger, childless, single and reporting more heterosexual partners in the last year.

Conclusions There was relatively little 'shopping around' between different services, suggesting that choice of contraceptive providers ensures a range of needs is met for most people. While general practice is the most commonly used source of supplies, community contraceptive clinics are seeing those potentially at higher sexual health risk, particularly the young and those with multiple partners. Ways of improving young people's access to services for contraceptive supplies need to be addressed.

Keywords contraceptive service use, Natsal-2, sexual behaviour, sexual health

J Fam Plann Reprod Health Care 2009; 35(1): 9-14

(Accepted 19 May 2008)

\section{Key message points}

- This study provides estimates of the proportion of the population accessing different types of contraceptive service in the last year and variation in this proportion by demographic and sexual behavioural characteristics.

- Women most commonly reported using general practice for contraceptive supplies and men most commonly reported using retail services. A minority of those accessing services used more than one type of source.

- Community contraceptive clinics are seeing those potentially at higher sexual health risk.

Despite concerns as to whether existing contraceptive services are both appropriate and sufficient to meet the contraceptive needs of men and women, ${ }^{2}$ there is little information at a population level on how use, and the profile of users, varies across services. Methods of routinely collecting data vary between local authorities and clinics. ${ }^{3}$ KT31 returns from National Health Service (NHS) community contraceptive services and from Brook Advisory Centres do provide national data on the number of attendees, the age and sex of attendees, and the contraceptive method provided. However, the validity of the data from these sources has never been independently assessed. Much research is conducted within the health service, excluding non-users; and though research has been conducted in community contraceptive services, little is known about general practice, and less about retail outlets. Selection bias has therefore been an important limitation of much research to date.

The main questions we address in this paper are: which contraceptive services are most commonly used; which service types are more commonly used in conjunction with other services; how the demographic and sexual behaviour characteristics of users differ between services, and what is the prevalence of non-use and who are the non-users? A better understanding of the characteristics of the users of different 
services will help with the planning and development of contraceptive provision in the UK.

\section{Methods}

The second National Survey of Sexual Attitudes and Lifestyles ('Natsal-2') was conducted in 1999-2001. Further details on the survey methods have been described elsewhere. ${ }^{4,5}$ In brief, a probability sample of 16-44-yearolds in Britain were interviewed about their sexual attitudes and behaviours. Data were collected by a combination of computer-assisted personal interviews (CAPI) and computerassisted self-interviews (CASI). A total of 11161 individuals participated in the study (4762 men and 6399 women). The overall response rate was $65.4 \% .4,6$ For the purpose of examining the profile of those most likely to be in need of a contraceptive service, individuals reporting the following characteristics were excluded from this analysis:

(1) exclusive use of male or female sterilisation in the last year (men and women who reported that they or their partner had been sterilised, but had used another other contraceptive methods in the last year, were not excluded from the sample),

(2) medical investigations for infertility,

(3) only same-sex partners in the last year, and

(4) no vaginal intercourse in the last year.

Women who reported that they were pregnant were retained in the sample as it was not possible to determine whether or not the pregnancy was planned.

Respondents were asked to identify sources of contraceptive advice or supplies that they had accessed in the year prior to interview. Use of emergency hospital departments was a response in the survey, but was not included in our analysis. Very few respondents $(n=45)$ reported this as a source of contraceptive advice or supplies, and of those who did, all had used one of the other service categories. Contraceptive services were grouped into four broad categories: general practice [including own general practitioner (GP) or another GP at own surgery or other health centre], community contraceptive clinics (including local health authority clinic or sexual health/contraceptive service for young people), retail (including chemist/pharmacy, other types of shop, vending machines or mail order) and non-use of any service. We explored how use of these types of service varied by sociodemographic characteristics (age, ethnicity, educational attainment, social class, marital status, parity and area type) and the reported number of heterosexual partners in the last year. It is

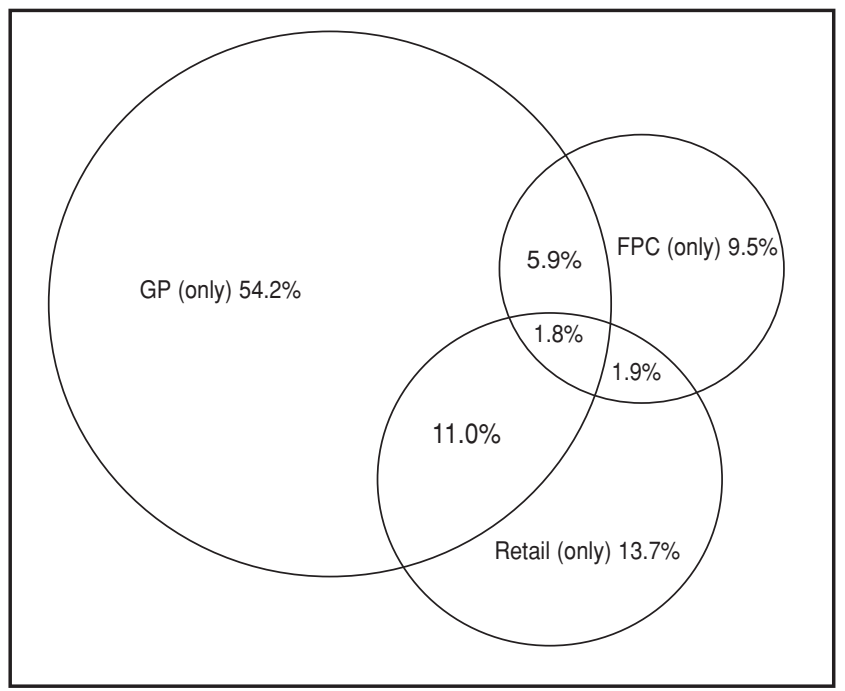

Figure 1 Proportional Venn diagram showing service use for contraceptive supplies and/or advice in the past year: women (denominator: service users only). FPC, family planning clinic; GP, general practitioner important to note that the (row) percentages shown in Tables 1 and 2 sum to more than $100 \%$ because respondents could report use of more than one type of service in the past year, thus 'types of service' are not mutually exclusive categories. Venn diagrams were created to provide a proportional representation of women and men's service use (see Figures 1 and 2$).^{7}$ As these diagrams only apply to those who have accessed services in the previous year, the percentages are different to those presented in the tables, where the denominator includes non-users.

All analyses were performed using the survey functions of STATA Version 7.0 to account for stratification, clustering and weighting of the sample. ${ }^{8}$ The data were weighted to correct for unequal selection probabilities and to match the age/sex population profile. 3,5 Values of $p<0.05$ are considered to be statistically significant for all analyses.

\section{Results}

\section{Sample characteristics}

After exclusions, the sample comprised 3369 men (70.7\% of all men interviewed) and 4375 women (68.4\% of all women interviewed).

\section{Women's use of contraceptive services}

General practice was the most commonly cited provider for contraceptive supplies with $59.2 \%$ (95\% CI $57.5 \%$ to $60.8 \%$ ) of sexually active women reporting use of this service in the last year (Table 1). Three-quarters (74.9\%) of this group reported exclusive use of general practice. Retail sources were used by $22.5 \%$ (95\% CI $21.1 \%$ to $24.0 \%$ ) of the women, with nearly half $(48.3 \%)$ of retail users reporting that this was their sole source of supplies. Fifteen percent $(95 \%$ CI $13.8 \%$ to $16.3 \%$ ) of the women reported use of community contraceptive clinics, and again half of these reported exclusive use of this service (49.3\%). Overall, $16.3 \%$ (95\% CI $15.1 \%$ to $17.6 \%$ ) of women reported using more than one category of service in the past year. Figure 1 shows that the largest overlap in service use was between general practice and retail, with over one in ten $(11.0 \%)$ of the women reporting any service use accessing both these services in the last year. One in five $(20.7,95 \%$ CI $19.4 \%$ to $22.0 \%$ ) sexually active women reported that they had not accessed a service for contraceptive supplies in the last year.

Variations in women's use of specific services by demographic and sexual behaviour characteristics are

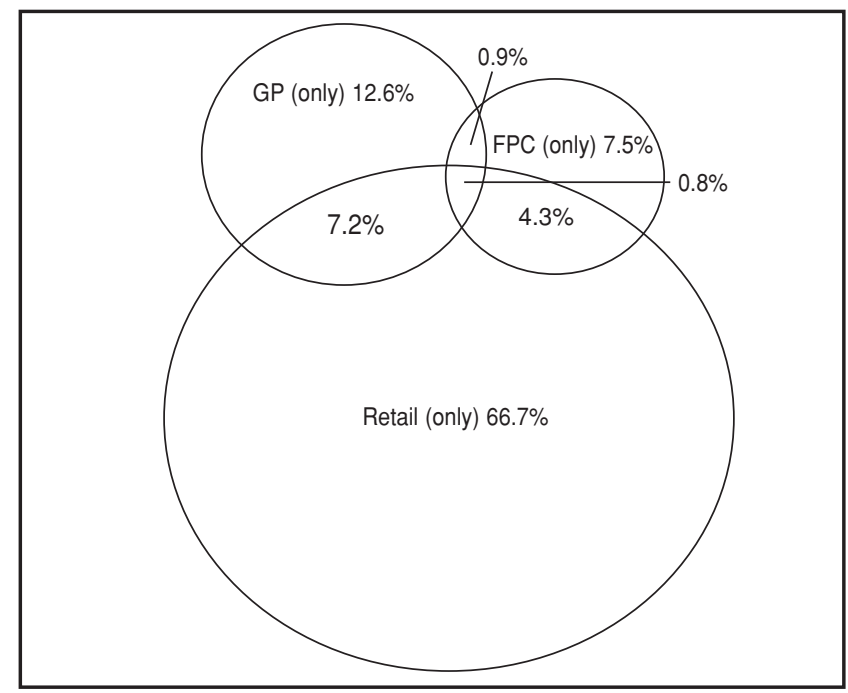

Figure 2 Proportional Venn diagram showing service use for contraceptive supplies and/or advice in the past year: men (denominator: service users only). FPC, family planning clinic; GP, general practitioner 
Table 1 Variations in the prevalence $(95 \% \mathrm{Cl})$ of using selected sources for contraceptive advice/supplies in the past year by selected sociodemographic and sexual behaviour characteristics: womena

\begin{tabular}{|c|c|c|c|c|c|c|}
\hline \multirow[t]{2}{*}{ Characteristic } & \multirow[t]{2}{*}{ Denominatorb } & \multicolumn{5}{|c|}{ Type of contraceptive source in the past yearc } \\
\hline & & $\begin{array}{l}\text { General } \\
\text { practice }\end{array}$ & $\begin{array}{l}\text { Community } \\
\text { clinics }^{d}\end{array}$ & Retaile & $\begin{array}{l}\text { More than one } \\
\text { source }\end{array}$ & None \\
\hline All women & $4375 / 3755$ & $59.2(57.5-60.8)$ & $15.0(13.8-16.3)$ & $22.5(21.1-24.0)$ & $16.3(15.1-17.6)$ & $20.7(19.4-22.0)$ \\
\hline $\begin{array}{l}\text { Sociodemographic } \\
\text { Age (years) } \\
16-17 \\
18-19 \\
20-24 \\
25-29 \\
30-34 \\
35-44\end{array}$ & $\begin{array}{c}147 \text { / } 170 \\
237 / 248 \\
688 \text { / } 658 \\
967 / 804 \\
993 / 792 \\
1343 / 1083\end{array}$ & $\begin{array}{l}p<0.0001 \\
54.0(45.2-62.5) \\
72.6(65.9-78.4) \\
72.8(68.7-76.4) \\
69.0(65.5-72.2) \\
57.0(53.6-60.3) \\
43.0(40.0-46.0)\end{array}$ & $\begin{array}{l}p<0.0001 \\
33.7(25.9-33.7) \\
28.6(22.6-35.4) \\
23.8(20.1-27.8) \\
12.6(10.4-15.1) \\
10.7 \quad(8.8-13.1) \\
8.6 \quad(7.1-16.3)\end{array}$ & $\begin{array}{l}p=0.4433 \\
21.6(15.1-30.2) \\
24.9(19.0-32.0) \\
23.1(19.5-27.1) \\
21.9(19.0-25.1) \\
24.8(21.8-28.0) \\
20.5(18.1-23.0)\end{array}$ & $\begin{array}{l}p<0.0001 \\
19.7(13.6-27.5) \\
30.2(24.0-37.2) \\
26.3(22.7-30.2) \\
17.0(14.5-19.8) \\
14.2(12.0-16.7) \\
7.5(6.2-9.1)\end{array}$ & $\begin{aligned} & p<0.0001 \\
& 13.8(8.7-21.2) \\
& 8.0(5.1-12.4) \\
& 8.9(6.9-11.5) \\
& 13.8(11.4-16.7) \\
& 21.8(19.1-24.7) \\
& 36.0(33.2-38.9)\end{aligned}$ \\
\hline $\begin{array}{l}\text { Ethnicity } \\
\text { White } \\
\text { Black Caribbean } \\
\text { Black African } \\
\text { Indian } \\
\text { Pakistani } \\
\text { Other }\end{array}$ & $\begin{array}{c}3938 \text { / } 3376 \\
107 / 93 \\
69 / 70 \\
71 / 58 \\
40 / 32 \\
131 / 111\end{array}$ & $\begin{array}{l}p=0.1531 \\
59.3(57.6-61.0) \\
62.1(51.7-71.5) \\
68.0(54.8-78.9) \\
54.2(40.8-67.0) \\
59.3(42.3-74.3) \\
48.3(39.0-57.4)\end{array}$ & $\begin{array}{l}p=0.0448 \\
14.3(13.1-15.6) \\
23.9(16.3-33.6) \\
22.5(13.2-35.7) \\
19.8(11.0-33.1) \\
18.4(8.4-35.6) \\
19.0(12.6-27.7)\end{array}$ & $\begin{array}{l}p=0.5370 \\
22.4(20.9-23.9) \\
28.7(19.5-40.0) \\
18.9(10.8-31.1) \\
23.7(14.6-36.0) \\
32.3(18.3-50.3) \\
21.3(14.6-29.9)\end{array}$ & $\begin{array}{l}p=0.1085 \\
15.9(14.7-17.2) \\
26.1(17.7-36.7) \\
23.8(14.4-36.6) \\
13.1(5.9-26.8) \\
19.6(9.2-37.0) \\
16.3(10.4-24.7)\end{array}$ & $\begin{array}{l}p=0.1899 \\
20.8(19.4-22.2) \\
15.1(9.0-24.2) \\
17.5(10.3-28.3) \\
14.6(6.7-28.8) \\
27.7(20.1-36.9) \\
20.7(19.4-22.0)\end{array}$ \\
\hline $\begin{array}{l}\text { Highest level of } \\
\text { educational qualification }\end{array}$ & & $p=0.0001$ & $p=0.0011$ & $p<0.0001$ & $p=0.379$ & $p<0.0001$ \\
\hline $\begin{array}{l}\text { Degree } \\
\text { A-level/equivalent } \\
\text { GCSE/equivalent } \\
\text { Other } \\
\text { None }\end{array}$ & $\begin{array}{c}953 / 743 \\
1190 / 1066 \\
1674 / 1490 \\
48 / 37 \\
497 / 404\end{array}$ & $\begin{array}{l}53.9(50.3-57.4) \\
60.7(57.5-63.8) \\
62.4(59.9-65.0) \\
42.5(27.4-59.1) \\
54.6(49.6-59.6)\end{array}$ & $\begin{array}{l}13.7(11.4-16.4) \\
17.3(15.0-19.9) \\
14.9(13.1-16.9) \\
31.7(17.8-49.8) \\
10.5(7.9-13.9)\end{array}$ & $\begin{array}{l}33.2(29.8-36.8) \\
24.1(21.4-27.0) \\
19.0(16.9-21.2) \\
21.2(10.4-38.4) \\
11.6(8.8-15.1)\end{array}$ & $\begin{array}{l}18.5(15.9-20.4) \\
17.8(15.4-20.4) \\
15.4(13.6-17.4) \\
19.4(8.6-28.3) \\
11.7 \quad(8.8-15.4)\end{array}$ & $\begin{array}{l}18.9(16.4-21.8) \\
17.0(14.8-19.4) \\
19.9(17.9-22.0) \\
22.3(12.5-36.6) \\
36.0(31.1-40.9)\end{array}$ \\
\hline $\begin{array}{l}\text { Social class } \\
\text { I/II/III } \\
\text { IV/V/unemployed }\end{array}$ & $\begin{array}{c}3054 \text { / } 2590 \\
840 / 730\end{array}$ & $\begin{array}{l}p=0.1442 \\
58.3(56.4-60.2) \\
61.4(57.7-65.0)\end{array}$ & $\begin{array}{l}p=0.0447 \\
15.2(13.8-16.7) \\
12.2(10.1-14.8)\end{array}$ & $\begin{array}{l}p=0.0002 \\
24.1(22.4-25.9) \\
16.9(14.1-20.1)\end{array}$ & $\begin{array}{l}p=0.5335 \\
16.4(14.9-17.9) \\
15.3(12.8-18.3)\end{array}$ & $\begin{array}{l}p=0.0010 \\
19.6(18.1-21.3) \\
25.4(22.3-28.7)\end{array}$ \\
\hline $\begin{array}{l}\text { Area type } \\
\text { Urban } \\
\text { Suburban } \\
\text { Rural }\end{array}$ & $\begin{array}{c}1207 / 847 \\
2511 / 2281 \\
657 / 627\end{array}$ & $\begin{array}{l}p=0.6578 \\
57.7(54.3-61.1) \\
59.6(57.4-61.8) \\
59.4(55.2-63.4)\end{array}$ & $\begin{array}{l}p<0.0001 \\
18.3(15.7-21.4) \\
15.5(14.0-17.2) \\
8.7(6.7-11.3)\end{array}$ & $\begin{array}{l}p=0.9553 \\
22.7(19.8-26.0) \\
22.3(20.5-24.2) \\
22.8(19.4-26.7)\end{array}$ & $\begin{array}{l}p=0.3137 \\
18.0(15.4-20.8) \\
16.0(14.5-17.7) \\
15.0(12.3-18.2)\end{array}$ & $\begin{array}{l}p=0.0860 \\
20.4(17.9-23.1) \\
19.8(18.2-21.6) \\
24.0(20.5-27.9)\end{array}$ \\
\hline $\begin{array}{l}\text { Marital status } \\
\text { Married/cohabiting } \\
\text { Previously married } \\
\text { Single, never married }\end{array}$ & $\begin{array}{c}2471 / 2394 \\
420 / 245 \\
1479 / 1113\end{array}$ & $\begin{array}{l}p<0.0001 \\
57.3(55.1-59.4) \\
52.7(47.4-58.0) \\
64.7(61.7-67.6)\end{array}$ & $\begin{array}{l}p<0.0001 \\
11.5(10.2-12.9) \\
11.1(8.2-14.7) \\
23.4(20.8-26.3)\end{array}$ & $\begin{array}{l}p=0.2695 \\
22.0(20.2-23.8) \\
20.4(16.6-24.9) \\
24.0(21.4-26.8)\end{array}$ & $\begin{array}{l}p<0.001 \\
13.4(12.0-14.9) \\
15.5(12.1-19.6) \\
22.8(20.3-25.5)\end{array}$ & $\begin{array}{l}p<0.0001 \\
23.0(21.3-24.8) \\
32.7(27.7-38.1) \\
13.0(11.1-15.0)\end{array}$ \\
\hline $\begin{array}{l}\text { Children }(n) \\
0 \\
1 \\
2+\end{array}$ & $\begin{array}{l}p<0.0001 \\
1817 / 1616 \\
905 / 733 \\
1653 / 1407\end{array}$ & $\begin{array}{l}p<0.0001 \\
63.4(60.8-66.0) \\
63.5(60.0-66.9) \\
52.0(49.3-54.6)\end{array}$ & $\begin{array}{l}p<0.0001 \\
20.8(18.7-23.1) \\
11.5 \quad(9.4-14.0) \\
10.1 \quad(8.7-11.8)\end{array}$ & $\begin{array}{l}p<0.001 \\
26.9(24.5-29.3) \\
19.7(17.0-22.8) \\
18.9(16.9-21.1)\end{array}$ & $\begin{array}{l}p<0.0001 \\
21.9(19.8-24.2) \\
14.5(12.1-17.3) \\
10.7(9.2-12.4)\end{array}$ & $\begin{array}{l}12.8(11.2-14.5) \\
20.1(17.4-23.2) \\
30.0(27.6-32.5)\end{array}$ \\
\hline $\begin{array}{l}\text { Sexual behaviour } \\
\text { Heterosexual partners } \\
\text { in past year }(n)\end{array}$ & & $p=0.0715$ & $p<0.0001$ & $p=0.0128$ & $p<0.001$ & $p<0.0001$ \\
\hline $\begin{array}{l}1 \\
2 \\
3-4 \\
5+\end{array}$ & $\begin{array}{l}3478 \text { / } 3061 \\
473 / 350 \\
220 / 177 \\
118 / 96\end{array}$ & $\begin{array}{l}58.7(56.8-60.5) \\
61.6(56.3-66.6) \\
66.8(59.1-73.7) \\
66.4(56.6-75.0)\end{array}$ & $\begin{array}{l}13.2(12.0-14.6) \\
20.6(16.5-25.5) \\
27.6(20.9-35.4) \\
31.5(22.4-42.3)\end{array}$ & $\begin{array}{l}21.8(20.3-23.4) \\
24.7(20.4-29.7) \\
25.6(19.3-33.2) \\
35.4(26.1-46.0)\end{array}$ & $\begin{array}{l}14.8(13.5-16.1) \\
20.0(15.9-24.9) \\
29.0(22.4-36.6) \\
34.9(25.3-45.9)\end{array}$ & $\begin{array}{rr}21.7 & (20.2-23.2) \\
15.7 & (12.5-19.5) \\
11.7 & (7.9-17.0) \\
8.7 & (4.8-14.9)\end{array}$ \\
\hline
\end{tabular}

aDenominator: service users and non-users.

bUnweighted / weighted denominators.

cThese categories are not mutually exclusive since respondents could report more than one source.

dCommunity clinics include mainstream community contraceptive clinics for all ages and designated young people's services.

eRetail includes pharmacies, vending machines, supermarkets, garages and postal delivery.

presented in Table 1. Strong associations were found with age and these were significant for all but retail outlets. A strong age gradient can be seen for community clinics, use of which decreased incrementally and significantly with age, with $33.7 \%$ of women aged 16-17 years reporting their use compared to $8.6 \%$ of women aged $35-44$ years. Age was also significantly associated with use of general practice; prevalence of use peaked among 18-24-year-olds. Nevertheless, the proportion of women visiting a general practice for contraceptive supplies was higher than the proportion visiting community contraceptive clinics for every age group. No significant differences in prevalence of attendance by ethnic group were seen in general practice, but use of community clinics was significantly higher amongst women from Black and minority ethnic groups than amongst those who were White. The proportion of women using retail outlets was significantly higher among women with higher levels of educational attainment, and amongst those in higher social class groups. There was little association between area type and use of a particular service, except for community contraceptive clinics where prevalence was highest amongst women living in urban areas $(18.3 \%$ vs $8.7 \%$ amongst those living in rural areas, $p<0.0001)$. A significant incremental increase was observed between the number of sexual partners in the last year and the use of community contraceptive clinics and retail outlets. Women with more than one sexual 
Table 2 Variations in the prevalence $(95 \% \mathrm{Cl})$ of using selected sources for contraceptive advice/supplies in the past year by selected sociodemographic and sexual behaviour characteristics: mena

\begin{tabular}{|c|c|c|c|c|c|c|}
\hline \multirow[t]{2}{*}{ Characteristic } & \multirow[t]{2}{*}{ Denominatorb } & \multicolumn{5}{|c|}{ Type of contraceptive source in the past yearc } \\
\hline & & $\begin{array}{l}\text { General } \\
\text { practice }\end{array}$ & $\begin{array}{l}\text { Community } \\
\text { clinics }^{d}\end{array}$ & Retaile & $\begin{array}{l}\text { More than one } \\
\text { source }\end{array}$ & None \\
\hline All men & 3369 / 4058 & $11.7(10.5-13.0)$ & 7.7 (6.7-8.7) & $42.7(40.8-44.5)$ & $7.3(6.3-8.4)$ & 45.1 (43.2-46.9) \\
\hline $\begin{array}{l}\text { Sociodemographic } \\
\text { Age (years) } \\
16-17 \\
18-19 \\
20-24 \\
25-29 \\
30-34 \\
35-44\end{array}$ & $\begin{array}{c}144 \text { / } 173 \\
206 \text { / } 230 \\
542 \text { / } 698 \\
658 \text { / } 830 \\
766 \text { / } 888 \\
1053 \text { / } 1240\end{array}$ & $\begin{array}{l}p=0.1226 \\
13.1 \quad(8.2-20.5) \\
16.0(10.8-23.1) \\
11.0(8.2-14.8) \\
8.9(6.8-11.7) \\
13.5(11.1-16.5) \\
11.5 \quad(9.6-13.9)\end{array}$ & $\begin{array}{lr}p<0.0001 \\
14.6 & (8.9-23.1) \\
20.3 & (14.6-27.4) \\
14.0 & (11.0-17.6) \\
5.5 & (3.9-7.8) \\
5.4 & (3.9-7.3) \\
3.9 & (2.8-8.7)\end{array}$ & $\begin{array}{l}p<0.0001 \\
51.8(41.7-61.7) \\
57.2(49.1-64.9) \\
59.5(54.4-64.4) \\
44.9(40.6-49.2) \\
37.0(33.5-40.7) \\
31.8(28.7-34.9)\end{array}$ & \begin{tabular}{rr}
\multicolumn{2}{c}{$p<0.0001$} \\
11.2 & $(6.6-18.6)$ \\
18.2 & $(12.4-25.7)$ \\
13.0 & $(9.8-17.1)$ \\
5.4 & $(3.7-7.9)$ \\
5.0 & $(3.7-7.9)$ \\
4.3 & $(3.2-5.9)$
\end{tabular} & $\begin{array}{l}p<0.0001 \\
31.2(22.6-41.3) \\
23.7(17.8-30.9) \\
27.8(23.7-32.2) \\
45.2(40.9-49.7) \\
50.2(46.4-54.1) \\
56.9(53.6-60.2)\end{array}$ \\
\hline $\begin{array}{l}\text { Ethnicity } \\
\text { White } \\
\text { Black Caribbean } \\
\text { Black African } \\
\text { Indian } \\
\text { Pakistani } \\
\text { Other }\end{array}$ & $\begin{array}{c}3064 \text { / } 3675 \\
84 \text { / } 103 \\
44 \text { / } 61 \\
64 \text { / } 78 \\
18 \text { / } 27 \\
84 \text { / } 102\end{array}$ & $\begin{aligned} & p=0.5150 \\
& 11.9(10.6-13.2) \\
& 5.2(2.0-12.6) \\
& 14.1(5.9-29.9) \\
& 8.1(3.4-18.1) \\
& 10.4(2.5-3.5) \\
& 13.9(7.5-24.3)\end{aligned}$ & $\begin{array}{l}p=0.2418 \\
7.9(6.9-9.1) \\
6.1(2.7-13.0) \\
1.1(0.2-7.5) \\
4.5(1.5-12.9) \\
14.9(3.2-46.6) \\
4.4(1.5-12.4)\end{array}$ & $\begin{array}{l}p=0.4583 \\
43.0(41.0-45.0) \\
33.8(23.6-45.7) \\
36.3(22.6-52.6) \\
39.0(26.8-52.7) \\
56.4(32.9-77.3) \\
41.6(31.2-52.8)\end{array}$ & $\begin{array}{l}p=0.3733 \\
7.6(6.5-8.7) \\
2.9(10.6-7.4) \\
1.1 \quad(0.2-7.5) \\
6.5(2.3-17.0) \\
10.9(1.6-47.9) \\
5.9(2.2-15.3)\end{array}$ & $\begin{array}{l}p=0.1299 \\
44.6(42.7-46.5) \\
57.8(45.6-69.2) \\
49.6(33.9-65.4) \\
55.1(41.2-68.3) \\
29.3(12.6-54.4) \\
45.1(33.9-56.8)\end{array}$ \\
\hline $\begin{array}{l}\text { Highest level of } \\
\text { educational qualification } \\
\text { Degree } \\
\text { A-level/equivalent } \\
\text { GCSE/equivalent } \\
\text { Other } \\
\text { None }\end{array}$ & $\begin{array}{c} \\
821 / 921 \\
1058 \text { / } 1294 \\
1113 \text { / } 1392 \\
29 \text { / 30 } \\
342 \text { / } 413\end{array}$ & $\begin{array}{ll}p=0.2989 \\
13.6 & (11.1-16.6) \\
11.1 & (9.1-13.4) \\
10.3 & (8.5-12.5) \\
14.5 & (4.6-37.7) \\
13.3 & (9.7-18.1)\end{array}$ & $\begin{array}{l}p=0.0610 \\
5.0(4.3-8.2) \\
8.1(6.5-10.2) \\
9.2(7.5-11.2) \\
4.5(0.9-19.4) \\
5.4 \quad(3.3-8.7)\end{array}$ & $\begin{array}{l}p<0.0001 \\
51.4(47.5-55.3) \\
44.0(40.7-47.3) \\
40.2(37.0-43.5) \\
30.2(15.6-50.4) \\
28.4(23.3-34.0)\end{array}$ & $\begin{array}{l}p=0.0531 \\
8.2(6.2-10.7) \\
8.2(6.4-10.4) \\
7.1 \quad(5.6-9.0) \\
3.5(0.5-21.0) \\
3.3 \quad(1.8-6.0)\end{array}$ & $\begin{array}{l}p<0.0001 \\
37.2(33.6-41.0) \\
45.0(41.7-48.2) \\
47.1(43.8-50.3) \\
54.2(34.2-72.9) \\
55.6(49.6-61.5)\end{array}$ \\
\hline $\begin{array}{l}\text { Social class } \\
\text { I/II/III } \\
\text { IV/V/unemployed }\end{array}$ & $\begin{array}{c}2531 / 3048 \\
623 \text { / } 754\end{array}$ & $\begin{array}{l}p=0.4150 \\
11.7(10.3-13.2) \\
13.0(10.3-16.2)\end{array}$ & $\begin{array}{l}p=0.1224 \\
6.5 \quad(5.5-7.6) \\
8.4(6.3-11.2)\end{array}$ & $\begin{array}{l}p=0.1658 \\
43.4(41.3-45.6) \\
39.9(35.6-44.3)\end{array}$ & $\begin{array}{ll}p=0.850 \\
7.3 & (6.1-8.6) \\
7.0 & (5.1-9.7)\end{array}$ & $\begin{array}{l}p=0.8043 \\
45.7(43.6-47.9) \\
45.1(40.7-49.6)\end{array}$ \\
\hline $\begin{array}{l}\text { Area type } \\
\text { Urban } \\
\text { Suburban } \\
\text { Rural }\end{array}$ & $\begin{array}{c}953 \text { / } 907 \\
1887 \text { / } 2438 \\
529 \text { / } 713\end{array}$ & $\begin{array}{l}p=0.1327 \\
12.1 \quad(9.5-15.2) \\
12.4(10.8-14.1) \\
8.9(6.6-11.9)\end{array}$ & $\begin{array}{l}p=0.0001 \\
11.8(9.4-14.7) \\
6.6 \quad(5.5-7.9) \\
6.1 \quad(4.3-8.6)\end{array}$ & $\begin{array}{l}p=0.2526 \\
43.5(39.6-47.5) \\
43.3(40.9-45.8) \\
39.2(35.0-43.6)\end{array}$ & 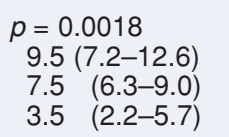 & $\begin{array}{l}p=0.0753 \\
41.9(38.1-45.9) \\
45.1(42.7-47.5) \\
49.0(44.4-53.6)\end{array}$ \\
\hline $\begin{array}{l}\text { Marital status } \\
\text { Married/cohabiting } \\
\text { Previously married } \\
\text { Single, never married }\end{array}$ & $\begin{array}{c}1640 / 2356 \\
223 / 168 \\
1501 / 1528\end{array}$ & $\begin{array}{l}p=0.0001 \\
13.9(12.2-15.7) \\
7.6 \quad(4.4-12.8) \\
8.7 \quad(7.1-10.7)\end{array}$ & $\begin{array}{l}p<0.0001 \\
5.4 \quad(4.4-6.6) \\
1.7 \quad(0.5-5.5) \\
11.8(10.0-14.0)\end{array}$ & $\begin{array}{l}p<0.0001 \\
32.7(30.3-35.1) \\
44.1(37.1-51.3) \\
57.9(54.8-61.0)\end{array}$ & $\begin{array}{l}p<0.0001 \\
5.9(4.8-7.2) \\
2.6(1.1-6.2) \\
10.0(8.2-12.2)\end{array}$ & $\begin{array}{l}p<0.0001 \\
53.8(51.3-56.4) \\
48.2(41.1-55.5) \\
31.2(28.5-34.0)\end{array}$ \\
\hline $\begin{array}{l}\text { Children }(n) \\
0 \\
1 \\
2+\end{array}$ & $\begin{array}{c}2301 \text { / } 2302 \\
500 \text { / } 631 \\
837 \text { / } 1125\end{array}$ & $\begin{array}{l}p=0.0001 \\
9.5 \quad(8.1-11.2) \\
12.3(9.6-15.7) \\
15.7(13.3-18.5)\end{array}$ & $\begin{array}{l}p=0.0017 \\
9.2(7.8-10.8) \\
6.4 \quad(4.5-9.0) \\
5.3 \quad(3.9-7.1)\end{array}$ & $\begin{array}{l}p<0.0001 \\
51.1(48.6-53.6) \\
35.3(31.0-39.2) \\
29.5(26.3-32.9)\end{array}$ & $\begin{array}{l}p=0.0024 \\
8.8(7.3-10.5) \\
5.8 \quad(4.0-8.3) \\
5.1 \quad(3.7-6.9)\end{array}$ & $\begin{array}{l}p<0.0001 \\
38.6(36.3-41.0) \\
51.6(46.9-56.3) \\
54.6(51.0-58.2)\end{array}$ \\
\hline $\begin{array}{l}\text { Sexual behaviour } \\
\text { Heterosexual partners } \\
\text { in past year }(n) \\
1 \\
2 \\
3-4 \\
5+\end{array}$ & $\begin{array}{c}2222 \text { / } 2827 \\
453 \text { / } 496 \\
362 \text { / } 386 \\
250 \text { / } 255\end{array}$ & $\begin{aligned} p=0.0662 & \\
12.7 & (11.2-14.3) \\
8.1 & (5.5-11.8) \\
11.4 & (8.0-16.1) \\
8.7 & (5.5-13.5)\end{aligned}$ & $\begin{array}{l}p=0.0008 \\
6.5(5.5-7.7) \\
8.6(6.0-12.2) \\
11.1(7.8-15.7) \\
13.8(9.4-19.8)\end{array}$ & $\begin{array}{l}p<0.0001 \\
37.4(35.2-39.6) \\
56.0(50.5-61.4) \\
54.3(48.3-60.1) \\
59.9(52.6-66.9)\end{array}$ & $\begin{array}{l}p=0.045 \\
6.6(5.5-7.9) \\
7.3(4.8-10.3) \\
10.0(6.8-14.4) \\
11.5(7.5-17.4)\end{array}$ & $\begin{array}{l}p<0.0001 \\
50.0(47.7-52.2) \\
34.6(29.9-39.7) \\
32.2(27.1-38.1) \\
28.5(22.4-35.5)\end{array}$ \\
\hline
\end{tabular}

aDenominator: service users and non-users.

bUnweighted / weighted denominators.

cThese categories are not mutually exclusive since respondents could report more than one source.

dCommunity clinics include mainstream community contraceptive clinics for all ages and designated young people's services.

eRetail includes pharmacies, vending machines, supermarkets, garages and postal delivery.

partner in the last year were more likely to have used more than one contraceptive service in the last year.

Overall, one in five $(20.7 \%)$ sexually active women had used no service in the past year to access contraceptive supplies. Non-use was highest among 35-44-year-olds $(36.0 \%)$ and lowest among 18-24-year-olds (8.7\%). Non-use was also higher amongst those with lower educational attainment, those of lower social class, those who were currently or previously married, and those with more than one child. In terms of sexual behaviour, non-use was higher amongst those with one partner, although nearly one in ten women $(8.7 \%)$ who reported five or more partners had not accessed a service in the last year.

\section{Men's use of contraceptive services}

Nearly half $(45.1 \%, 95 \%$ CI $43.2 \%$ to $46.9 \%)$ of all sexually active men reported that they had not accessed any services for contraceptive supplies in the last year (Table 2). Among those who reported that they had, the most common source of supplies was retail services, with $42.7 \%$ (95\% CI $40.8 \%$ to $44.5 \%$ ) having accessed this type of service, and $84.3 \%$ of this group reporting this was their only source of contraceptive supplies. General practice was cited by $11.7 \%$ (95\% CI $10.5 \%$ to $13.0 \%$ ) of male respondents (with $58.5 \%$ of general practice users reporting exclusive use) and community contraceptive clinics by $7.7 \%$ (95\% CI $6.7 \%$ to $8.7 \%$ ) (with $55.6 \%$ of community contraceptive clinic users 
reporting exclusive use). There was little overlap in use of different providers amongst the male service users (Figure 2), with just $7.3 \%$ of these men reported using more than one type of service. As seen amongst the women, the greatest overlap in service use was between general practice and retail, with $3.9 \%$ of men reporting use of both these types of services.

Age was significantly associated with use of community contraceptive clinics and retail outlets, but not with use of general practice (Table 2.). The proportion of men using community contraceptive clinics was highest in the 18-19year-old age range $(20.3 \%)$ The proportion of men using retail outlets was highest among those with a degree (51.4\%), with prevalence of use decreasing incrementally and significantly with decline in educational attainment (to $28.4 \%$ of men reporting no educational qualifications). No significant difference was observed between ethnicity and use of any of the types of contraceptive services. Use of general practice was significantly higher amongst married and cohabiting men and those with two or more children, while use of community contraceptive clinics and retail outlets was significantly more common amongst men who were single and those who did not have children. As observed among women, the proportion of men using community contraceptive clinics was significantly higher among those living in urban areas than those living in rural areas. Use of more than one service was highest amongst 18-19-year-olds, those living in urban areas, the single and those childless. Prevalence of use of community contraceptive clinics, retail services, and of use of more than one service, increased with numbers of heterosexual partners. This association was not observed amongst those using general practice.

The characteristics of men accessing no service for contraceptive supplies were similar to those of female nonusers. The prevalence of non-use was highest amongst men aged 35-44 years $(56.9 \%)$ and lowest amongst those aged $18-19$ years $(23.7 \%, p<0.0001)$. It was also higher amongst those with children compared to those without, and amongst those reporting no educational qualifications compared to those with. Although non-use was more common amongst those reporting one sexual partner in the last year compared to those who reported two or more, over a quarter of men with five or more partners $(28.5 \%)$ reported non-use.

\section{Ethical approval}

Ethical approval was obtained from University College Hospital, London School of Hygiene and Tropical Medicine ethics committee, North Thames Multicentre, and all local research ethics committees in Britain.

\section{Discussion}

General practice was the most frequently reported source of contraceptive advice and supplies for women, with nearly two-thirds of our sample reporting use in the last year. These findings are in line with other population-based surveys reporting on women's use of contraceptive services. ${ }^{9,10}$ Overall, three times as many women had accessed general practice for contraceptive supplies as had done so in community contraceptive clinics, and amongst women aged over 25 years, five times as many. More than half of 16-17year-olds did so, despite concerns that young people's fears around confidentiality may act as a barrier to general practice. ${ }^{11}$ The proportion of men who accessed contraceptive supplies from general practice was lower; little more that one in ten, even amongst those aged 18-19 years, with retail outlets the most frequently cited contraception source amongst men.

Over the last decade much debate has centred on whether or not different types of contraceptive services duplicate or complement each other. $^{2,12}$ We found relatively little 'shopping around' between contraceptive services. Only one in six women, and fewer men, reported using more than one service in the last year. It was not possible to determine whether use of additional services was due to consumer choice, or due to referrals made by providers who were unable to offer the service required. However, among the minority of those who used more than one service, use of an additional service was less common amongst women attending general practice for contraceptive supplies. Those who 'shopped around' were more likely to be younger, single and have more sexual partners.

Those with larger numbers of sexual partners were more likely to report using community contraceptive clinics and retail outlets for contraceptive advice and supplies. In contrast, general practice appears to be attracting older, monogamous women in the family-building stages of their lives. Our data suggest therefore that community contraceptive clinics are seeing those at higher sexual health risk. It is important to acknowledge that this is likely to be confounded by age and marital status. The bivariate analysis presented provides a profile of users (and non-users) of contraceptive services rather than a parsimonious model of risk factors. From a public health and service provision prospective it is important that the client profiles of different services are understood. Multivariate analysis is therefore not appropriate here. For example, by adjusting for age and number of sexual partners, the effect of partner numbers would be lost because of its association with age.

While these data cannot answer the question of whether different services are meeting people's needs, they illustrate that use does vary depending on individual demographic and sexual behavioural characteristics. The national Evaluation of One-Stop Shop Models of Sexual Health Provision found that some target groups at particular risk, such as young men and some people from ethnic minority groups, maintain a strong preference for specialist services. ${ }^{13}$ This supports the need for a range of contraceptive providers. A reduction in community contraceptive clinics could potentially have negative consequences for the most vulnerable. Women from Black and minority ethnic groups were more frequent users of community contraceptive clinics than were White women. However, the numbers representing different ethnic minority groups were too small to draw any strong conclusions about ethnicity and service use, and the categories derived for data analysis may be too broad to provide meaningful information about specific ethnic minority groups. Additionally, it is important to acknowledge that the availability and thus accessibility of community contraceptive clinics is often greater in urban centres, such that the observed association with ethnicity may, in part, reflect the greater density of ethnic minority groups in urban areas. ${ }^{14}$

It has been argued from a public health perspective that it is important to have choice to meet the needs of different users. Bardsley and colleagues comment that if provision of contraception through community contraceptive clinics was reduced, rather than accessing their general practice, many women would use less effective 'over the counter' methods or even no method at all. ${ }^{15}$ However, since the launch of the National Strategy for Sexual Health and HIV in 2001, there has been increasing emphasis on expanding the role of primary care in the provision of sexual health care in England, ${ }^{16}$ such that general practice may be becoming increasingly attractive to such women as a provider of contraception advice and supplies and, more generally, sexual and reproductive health services.

The results of this study may reflect patterns of contraceptive use. In this respect, a limitation of this study is that it is not possible to determine which methods were 
supplied by which service (although data on contraceptive use in the last year were collected by Natsal-2). Thus, it is not possible to ascertain whether contraceptive use was influenced by the service(s) attended and/or availability in a respondent's locality. For example, men may be more likely to be using retail outlets to obtain condoms if these are not available at their general practice. GPs are charged with providing a narrower range of contraceptive services and methods; in 2002 over $90 \%$ of GPs provided oral contraceptives and emergency contraception, only around half provided condoms and less than a third provided implants. ${ }^{17}$ Unfortunately, there is currently little incentive for GPs to increase the spectrum of methods available in general practice. There are only two Quality and Outcomes Framework (QOF) points directly applicable to contraception: (1) a written policy for responding to requests for emergency contraception and (2) a policy for providing pre-conceptual advice. ${ }^{18}$ Comprehensive contraceptive provision may even be limited in some of the specialist contraceptive services. The range of contraceptive methods within designated young people's services has been found to be often limited. 19

One in five sexually active women and nearly one in two sexually active men had accessed no contraceptive service in the past year. Since the numbers who were pregnant or planning pregnancy is unknown from Natsal-2 it was not possible to determine how many of these were people with unmet need in terms of service provision. However, it is worrying to find that $14 \%$ of sexually active young women and $31 \%$ of sexually active young men aged 16-17 years reported not accessing any type of contraceptive service. Stone and Ingham found in their survey of young people accessing youth-targeted sexual health services that less than one in three had accessed a sexual health service prior to first sexual intercourse. ${ }^{20}$ Considering the high rates of teenage pregnancy and sexually transmitted infections amongst young people, ${ }^{21,22}$ attention needs to be paid on how best to engage with this age group to ensure that their sexual and reproductive health needs are met.

Patterns of contraceptive use have changed over the last two decades. For example, pill use has declined slightly, while condom use has increased. ${ }^{23}$ Retail outlets are the most commonly reported source of contraceptive advice and supplies for men. Ways of providing support for men in these settings and linking men into health services need further exploration. Although this study found that almost half of men had not used any service for contraception in the past year, there is evidence that the number of men using contraceptive services is increasing. 9,10 Changing trends in contraceptive use over time, among women and men, are likely to have an impact on population needs in terms of service provision. A choice of contraceptive providers has the potential to lead to a greater proportion of women and men using effective and acceptable contraceptive methods. General practice is the setting providing contraceptive care to the majority of people in this country. In order for GPs and practice nurses to provide a comprehensive and quality contraceptive service, contractual incentives and training issues need to be addressed.

\section{Acknowledgements}

The authors thank the study participants, the team of interviewers and operations, and computing staff from the National Centre for Social Research who organised, carried out and edited the interviews and fieldwork.

Statements on funding and competing interests

Funding This study was supported by a grant from the Medical Research Council with funds from the Department of Health, the Scottish Executive and the National Assembly for Wales. The views expressed in this paper are those of the authors and do not necessarily reflect the views of the funding bodies.

Competing interests None identified.

\section{References}

1 Bone M. The Family Planning Services: Changes and Effects. London, UK: Her Majesty's Stationery Office, 1978.

2 French RS, Coope CM, Graham A, Gerressu M, Salisbury C, Stephenson JM; One-Stop Shop Evaluation Team. One stop shop versus collaborative integration: what is the best way of delivering sexual health services? Sex Transm Infect 2006; 82: 202-206.

3 Simms I, Nicoll A. Sexual Health in England: A Guide to National and Local Monitoring Surveillance and Monitoring Data. National HIV Prevention Information Service. Briefing 3. London, UK: Health Education Authority, 2000.

4 Johnson AM, Mercer CH, Erens B, Copas AJ, McManus S, Wellings $\mathrm{K}$, et al. Sexual behaviour in Britain: partnerships, practices, and HIV risk behaviours. Lancet 2001; 358: 1835-1842.

5 Johnson AM, Wadsworth J, Wellings K, Field J. Sexual Attitudes and Lifestyles. Oxford, UK: Blackwell Scientific, 1994.

6 Erens, B, McManus S, Field J, Korovessis C, Johnson AM, Fenton, KA. National Survey of Sexual Attitudes and Lifestyles II: Technical Report. London, UK: National Centre for Social Research, 2001

7 Chow S, Rodgers P. Constructing area-proportional Venn and Euler diagrams with three circles. Extended abstract presented at the Euler Diagrams Workshop, Paris, France, 2005. http://www.cs.kent.ac.uk/people/staff/pjr/EulerVennCircles/Eul erVennApplet.html [Accessed 4 November 2007].

8 StataCorp. Stata Statistical Software: Release 7.0. College Station, TX: Stata Corporation, 2001.

9 O'Sullivan I, Keyse L, Park N, Diaper A, Short S. Contraception and Sexual Health. 2004/5. London, UK: HMSO, 2005.

10 French RS, Mercer CH, Kane R, Kingori P, Stephenson JM, Wilkinson P, et al. What impact has England's Teenage Pregnancy Strategy had on young people's knowledge of and access to health services for contraceptive advice and supplies? Findings from the Teenage Pregnancy Strategy Evaluation. J Adolesc Health 2007; 41: 594-601.

11 Jacobson L, Richardson G, Parry-Langdon N, Donovan C. How do teenagers and primary healthcare providers view each other? An overview of key themes. Br J Gen Pract 2001; 51: 811-816.

12 Singleton CD, Reuter S. Dual provision or duplication? A survey of family planning provision. Br J Fam Plann 1999; 25: 41-44.

13 One-Stop Shop Evaluation Team. Evaluation of OneStop Shop Models of Sexual Health Provision. Executive Summary. 2007. http://www.dh.gov.uk/en/Publicationsand statistics/Publications/PublicationsStatistics/DH_083200 [Accessed 4 November 2007].

14 Tinsley J, Jacobs M. Deprivation and ethnicity in England: a regional perspective. Regional Trends 2006; 39. http://www.statistics.gov.uk/articles/RegionalTrends/Article3RT 39.pdf [Accessed 4 November 2007].

15 Bardsley M, Newman M, Morgan D, Jabobsen B. Estimating the balance of general practice versus family planning clinic coverage of contraception services in London. B J Fam Plann 2000; 26: 21-25.

16 Department of Health. The National Strategy for Sexual Health and HIV. London, UK: HMSO, 2001.

17 Teenage Pregnancy Strategy Evaluation Team. An Audit of Contraceptive Provision in General Practice. Internal briefing document No. 8. London, UK: Teenage Pregnancy Unit, 2003.

18 Department of Health. Quality and Outcomes Framework. Guidance - Updated August 2004. http://www.dh.gov.uk/en/ Policyandguidance/Organisationpolicy/Primarycare/Primarycar econtracting/QOF/DH 4125653 [Accessed 4 November 2007].

19 Teenage Pregnancy Strategy Evaluation Team. An Audit of Contraceptive Service Provision in England, with Special Reference to Service Provision for Young People. Internal briefing document No. 7. London, UK: Teenage Pregnancy Unit, 2002.

20 Stone N, Ingham R. When and why do young people in the United Kingdom first use sexual health services? Perspect Sex Reprod Health 2003; 35: 114-120.

21 Wilkinson P, French R, Kane R, Lachowycz K, Stephenson J, Grundy $\mathrm{C}$, et al. Teenage conceptions, abortions, and births in England, 1994-2003, and the national teenage pregnancy strategy. Lancet 2006; 368: 1879-1886.

22 The UK Collaborative Group for HIV and STI Surveillance. Testing Times. HIV and Other Sexually Transmitted Infections in the United Kingdom: 2007. London, UK: Health Protection Agency Centre for Infections, November 2007.

23 Office for National Statistics. Trends in Fertility and Contraception in the Last Quarter of the 20th Century. Population Trends (100), Summer 2000. 